\section{ASSISTÊNCIA PRESTADA À ADOLESCENTE NO MOMENTO DO PARTO EM UMA MATERNIDADE DE ALTO RISCO}

\author{
Assistance provided to adolescents at childbirth in a high-risk \\ maternity hospital
}

\author{
Atención al adolescente en el momento del parto en una \\ maternidad de alto riesgo
}

\section{RESUMO}

Objetivo: Analisar as práticas obstétricas realizadas em adolescentes parturientes atendidas em uma maternidade de alto risco. Métodos: Estudo observacional, de corte transversal, documental e descritivo, realizado com busca em 157 prontuários de parturientes adolescentes atendidas em maternidade de alto risco em Maceió-AL, no período de janeiro a junho/2013. Avaliaram-se variáveis socioeconômicas, práticas obstétricas e clínica, sendo os resultados apresentados em frequência simples. Resultados: Entre as parturientes, a idade variou de 14 a 19 anos, e $96(61,1 \%)$ viviam em união estável. Com relação aos dados obstétricos, 125 (79,6\%) eram primigestas e 73 realizaram de 4 a 6 consultas de pré-natal. Em $107(68,1 \%)$ prontuários o partograma não foi encontrado. Entre os partos realizados, em $75(47,8 \%)$ os profissionais não realizaram episiotomia, em $110(70,1 \%)$ foram realizadas manobras ativas do $3^{\circ}$ estágio e $146(86,6 \%)$ adolescentes não tiveram complicações clínicas no parto. Conclusão: Evidenciou-se que algumas práticas obstétricas preconizadas pelo Ministério da Saúde estão sendo realizadas com as parturientes adolescentes, mas não são suficientes para uma assistência de qualidade.

Descritores: Adolescência; Gravidez; Parto; Humanização; Enfermagem.

\section{ABSTRACT}

Objective: To examine the obstetric practices carried out in parturient adolescents seen in a high-risk maternity hospital. Methods: Observational, cross-sectional, documental, and descriptive study, performed through search in 157 medical records of parturient adolescents seen in a high-risk maternity in Maceió, AL, Brazil, in the period from January to June 2013. Socioeconomic variables and the obstetric and clinic practices were evaluated, and the results were presented in simple frequency. Results: Among the parturient adolescents, age ranged from 14 to 19 years, and 96 (61.1\%) were in a stable relationship. Regarding the obstetric data, 125 (79.6\%) were primigravidae, and 73 attended between 4 and 6 prenatal visits. In 107 (68.1\%) medical records, the partogram was not found. Of the childbirths, in 75 (47.8\%) the professionals did not perform episiotomy, in 110 (70.1\%) active management of the 3rd stage of labour was practiced, and 146 (86.6\%) adolescents did not have complications during childbirth. Conclusion: The study evidenced that some obstetric practices recommended by the Ministry of Health are being performed with the parturient adolescents, but are not enough for quality care.

Descriptors: Adolescence, Pregnancy, Childbirth, Humanization, Nursing.
Artigo Original

Márcia Juliana Mello da Silva ${ }^{(1)}$ Maria Elisângela Torres de Lima Sanches $^{(1)}$

Amuzza Aylla Pereira dos Santos $^{(1)}$

Juliana Bento de Lima Holanda ${ }^{(1)}$ Mariana Sarmento Santos ${ }^{(1)}$

1) Universidade Federal de Alagoas - UFAL - Maceió (AL) - Brasil

Recebido em: 27/08/2014 Revisado em: 16/11/2014 Aceito em: 18/01/2015 


\section{RESUMEN}

Objetivo: Analizar las prácticas obstétricas realizadas en adolescentes parturientas asistidas en una maternidad de alto riesgo. Métodos: Estudio observacional, de corte transversal, documental y descriptivo realizado con una búsqueda en 157 historiales clínicos de parturientas adolescentes asistidas en una maternidad de alto riesgo de Maceió-AL, en el periodo entre enero y junio/2013. Se evaluaron las variables socioeconómicas, prácticas obstétricas y clínica con los resultados presentados en frecuencia simples. Resultados: La edad de las parturientas varió entre 14 y 19 años y $96(61,1 \%)$ vivían en unión estable. Sobre a los datos obstétricos, $125(79,6 \%)$ eran primigestas y 73 realizaron entre 4 y 6 consultas de prenatal. El partograma no ha sido encontrado en 107 (68,1\%) historiales clínicos. En 75 (47,8\%) de los partos realizados los profesionales no hicieron la episiotomía, en $110(70,1 \%)$ fueron realizadas maniobras activas del $3^{\circ}$ nivel y $146(86,6 \%)$ adolescentes no tuvieron complicaciones clínicas del parto. Conclusión: Se evidenció la realización de algunas prácticas obstétricas establecidas por el Ministerio de la Salud en las parturientas adolescentes pero las mismas no son suficientes para una atención de calidad.

Descriptores: Adolescente; Embarazo; Parto; Humanización de la atención; Enfermería.

\section{INTRODUÇÃO}

A adolescência é o período em que ocorrem as mudanças corporais e psicossociais do ser humano, é o período de transição entre a infância e a idade adulta ${ }^{(1)}$. A Organização Mundial de Saúde (OMS) restringe a adolescência à segunda década da vida (de 10 a 19 anos) e considera que a juventude se estende dos 15 aos 24 anos. Já o Estatuto da Criança e do Adolescente (ECA) considera adolescente a faixa etária de 12 a 18 anos $^{(2)}$.

Agravidez na adolescência preocupa os diversos setores da sociedade, pois cerca de 14 milhões de adolescentes, de 15 a 19 anos, tornam-se mães a cada ano, perfazendo um total de mais de $10 \%$ dos nascimentos no mundo ${ }^{(3,4)}$. No Brasil, houve uma redução de $30 \%$ no número de partos em adolescentes na faixa etária de 15 a 19 anos, porém, a faixa etária de 10 a 15 anos permanece inalterada, apresentando o número de 27 mil partos a cada ano, o que representa $1 \%$ do total de partos no país ${ }^{(5)}$.

A OMS afirma que a gravidez e o parto na adolescência estão associados a riscos mais elevados para a saúde materna, pois complicações da gravidez e do parto são as principais causas de morte em adolescentes de 15 a 19 anos nos países em desenvolvimento ${ }^{(6)}$. Em contrapartida, o Ministério da Saúde (MS) enfatiza que a adolescência em si não é fator de risco para a gestação. Há, todavia, possibilidade de risco psicossocial, associado à aceitação ou não da gravidez $z^{(7)}$.
Tratando-se de estudos voltados para essa temática, a maioria aborda apenas os riscos físicos, psicológicos e sociais das condições na vida dos adolescentes, sem enfatizar os aspectos relacionados com a assistência no ciclo gravídico-puerperal. A atenção adequada no momento do parto representa uma atitude importante para amenizar os agravos que podem acompanhar a gravidez na adolescência $^{(8)}$.

Com a intenção de diminuir as práticas desnecessárias durante a assistência ao parto, a OMS publicou o Manual de Assistência ao Parto Normal: Um Guia Prático, em 1996, como referência para a implantação do parto humanizado nos serviços de saúde. Esse manual recomenda as práticas obstétricas vigentes, com base em evidências científicas ${ }^{(9,10)}$.

Essas práticas são classificadas em quatro categorias: categoria A - práticas claramente úteis e que carecem de ser incentivadas; categoria B - práticas prejudiciais ou ineficazes e que precisam ser eliminadas; categoria $\mathrm{C}$ - práticas com evidência insuficiente para apoiar uma recomendação e que necessitam ser usadas com precaução; categoria $\mathrm{D}$ - práticas frequentemente utilizadas de forma inapropriada, provocando mais danos que benefícios ${ }^{(11)}$.

No ano 2000, o Ministério da Saúde, baseado nas recomendações sobre boas práticas obstétricas, instituiu o Programa de Humanização no Pré-Natal e Nascimento (PHPN), cujo objetivo foi assegurar a melhoria do acesso da cobertura e da qualidade do acompanhamento pré-natal, da assistência ao parto e puerpério ${ }^{(12)}$.

Com isso, apesar de o PHPN ser uma política de atenção voltada para a qualificação da assistência ao prénatal e parto, falha por não considerar as especificidades da parturiente adolescente. Somando a isso, sabe-se que a gestante adolescente possui especificidade fisiológica e emocional, por isso, necessitam de assistência e cuidado exclusivos para sua faixa etária, principalmente no momento do parto ${ }^{(13,14)}$.

Diante do exposto, o objetivo deste estudo foi avaliar a assistência prestada às parturientes adolescentes atendidas em uma maternidade de alto risco em Maceió-AL.

\section{MÉTODOS}

Estudo documental, de corte transversal, descritivo, retrospectivo, com abordagem quantitativa, realizado na Maternidade Escola Santa Mônica (MESM), situada na cidade de Maceió-AL. A MESM é uma maternidade pública estadual de referência, que desenvolve atividades de assistência (de alta e média complexidade) e ensino, atendendo à comunidade da cidade de Maceió, assim como a todo o estado de Alagoas.

Foram avaliados os prontuários de todas as adolescentes $(\mathrm{n}=157)$ com idade entre 10 e 19 anos que tiveram parto 
normal e foram internadas na referida maternidade no período de janeiro/2013 a junho/2013. Foram excluídos os prontuários clínicos das adolescentes internadas na maternidade que chegaram em período expulsivo do parto, que tiveram parto na triagem, as com distúrbios mentais e com urgências obstétricas.

Acoleta ocorreuno Serviço de Arquivo Médico(SAME), utilizando-se formulário elaborado especificamente para este estudo, analisando-se as seguintes variáveis: características sociodemográficas, história obstétrica, boas práticas obstétricas utilizadas (uso de partograma, episitomia, ocitocina, manobras ativas no terceiro estágio de parto, amniotomia, contado do recém-nascido com a mãe após o nascimento e métodos não farmacológicos para o alivio da dor), além das características materna e neonatal das parturientes adolescentes.

A análise dos dados deu-se de forma descritiva, com a apresentação de número absoluto e frequência simples.

O estudo seguiu a Resolução 466/12 do Conselho Nacional de Saúde sobre pesquisa com seres humanos e os dados somente foram coletados após aprovação do projeto pelo Comitê de Ética, baseado no processo $\mathrm{n}^{\circ}$ 228572113.0.0000.5013, seguido da autorização da maternidade.

Tabela I - Dados sociodemográficos das adolescentes parturientes. Maceió-AL, 2013.

\begin{tabular}{|c|c|c|}
\hline Características & $\mathbf{n}$ & $\%$ \\
\hline \multicolumn{3}{|l|}{ Idade } \\
\hline 19 anos & 28 & 17,84 \\
\hline 18 anos & 40 & 25,48 \\
\hline 17 anos & 40 & 25,48 \\
\hline 16 anos & 28 & 17,83 \\
\hline 15 anos & 17 & 10,82 \\
\hline 14 anos & 4 & 2,55 \\
\hline \multicolumn{3}{|l|}{ Procedência } \\
\hline Maceió & 67 & 42,68 \\
\hline Outra cidade & 87 & 55,41 \\
\hline Não registrado & 3 & 1,91 \\
\hline \multicolumn{3}{|l|}{ Cor/raça } \\
\hline Branca & 5 & 3,19 \\
\hline Amarela & - & - \\
\hline Preta & 4 & 2,55 \\
\hline Indígena & - & - \\
\hline Parda & 144 & 91,71 \\
\hline Outra & - & - \\
\hline Não registrado & 4 & 2,55 \\
\hline \multicolumn{3}{|l|}{ Estado Civil } \\
\hline Solteira & 43 & 27,39 \\
\hline Casada & 13 & 8,29 \\
\hline União estável & 96 & 61,14 \\
\hline Outros & - & - \\
\hline Não registrado & 5 & 3,18 \\
\hline \multicolumn{3}{|l|}{ Anos de Estudo } \\
\hline Nenhum & 4 & 2,52 \\
\hline 1 a 4 & 28 & 17,84 \\
\hline 4 a 7 & 78 & 49,70 \\
\hline 11 ou mais & 40 & 25,48 \\
\hline Não registrado & 7 & 4,46 \\
\hline
\end{tabular}


Tabela II - Antecedentes obstétricos e características da gestação atual. Maceió-AL, 2013.

\begin{tabular}{|c|c|c|}
\hline Características & n & $\%$ \\
\hline \multicolumn{3}{|c|}{ Gestação da paciente } \\
\hline Primigesta & 125 & 79,62 \\
\hline Secundigesta & 22 & 14,01 \\
\hline Tercigesta & 7 & 4,45 \\
\hline Multigesta & 0 & 0 \\
\hline Não registrado & 3 & 1,92 \\
\hline \multicolumn{3}{|c|}{ Paridade da paciente } \\
\hline Primípara & 134 & 85,35 \\
\hline Paucípara & 20 & 12,74 \\
\hline Multípara & 0 & 0 \\
\hline Não registrado & 3 & 1,91 \\
\hline \multirow{2}{*}{\multicolumn{3}{|c|}{$\begin{array}{l}\text { Realização de consultas no } \\
\text { pré-natal }\end{array}$}} \\
\hline & & \\
\hline Nenhuma & 4 & 2,55 \\
\hline 1 a 3 & 26 & 16,56 \\
\hline 4 a 6 & 73 & 46,49 \\
\hline 7 ou mais & 37 & 23,57 \\
\hline Não registrado & 17 & 10,83 \\
\hline \multicolumn{3}{|c|}{ Idade gestacional no parto } \\
\hline$<20$ semanas & 0 & 0 \\
\hline 20 a 30 semanas & 12 & 7,79 \\
\hline 30 a 36 semanas & 78 & 50,65 \\
\hline 37 a 42 semanas & 64 & 41,56 \\
\hline
\end{tabular}

\section{RESULTADOS}

As pacientes avaliadas apresentaram idade variando entre 14 e 19 anos, com maior prevalência entre 17 e 18 anos $(59,9 \%)$. A maioria das parturientes era procedente do interior do estado $(87 ; 55,4 \%)$, considerava-se de etnia parda $(144 ; 91,7 \%)$, vivia em união estável $(96 ; 61,1 \%)$ e tinha de 4 a 7 anos de estudo $(78 ; 49,7 \%$ ) (Tabela I).

Com relação aos antecedentes obstétricos, 125 $(79,6 \%)$ adolescentes eram primigestas e $134(85,3 \%)$ eram primíparas. Sobre as características da gestação atual, 73 $(46,4 \%)$ realizaram de 4 a 6 consultas de pré-natal e 78 $(50,6 \%)$ tinham idade gestacional entre 30 e 36 semanas no momento do parto (Tabela II).

No que diz respeito às boas práticas obstétricas utilizadas nas adolescentes, 107 (68,1\%) prontuários avaliados não apresentaram o partograma e $18(11,5 \%)$ foram preenchidos incompletamente. Entre os partos realizados, em 75 (47,8\%) deles os profissionais não realizaram episiotomia e em 110 $(70,1 \%)$ foram realizadas manobras ativas do $3^{\circ}$ estágio, tais como administração de duas ampolas IM, tração controlada de cordão e massagem uterina. Com relação à realização da amniotomia, 150 (95,5\%) não tinham registro de dados nos prontuários, e destes, $134(85,7 \%)$ apresentavam líquido claro.

Com relação ao uso de ocitocina, em 127 (80,9\%) participantes essa conduta foi realizada, destes, em 21 $(13,3 \%)$ o uso ocorreu no primeiro estágio do parto. No que se refere ao contato pele a pele após o nascimento, em $152(96,8 \%)$ prontuários não havia registro dessa prática. De acordo com os dados coletados sobre a utilização de métodos não farmacológicos para alívio da dor, 150 (95,5\%) não foram registrados no prontuário (Tabela III).

Entre as adolescentes avaliadas, as características clínicas e perinatais indicaram que $136(86,6 \%)$ não tiveram complicações clínicas durante o parto e puerpério, a complicação de maior destaque foi delivramento incompleto $(112 ; 71,4 \%), 117(74,5 \%)$ ficaram dias internadas na instituição e $112(71,3 \%)$ dos recém-nascidos foram para o Alojamento Conjunto (ALCON) (Tabela IV). 
Tabela III - Boas práticas obstétricas utilizadas nas adolescentes. Maceió-AL, 2013.

\begin{tabular}{|c|c|c|}
\hline Característica & $\mathbf{n}$ & $\%$ \\
\hline \multicolumn{3}{|c|}{ Utilização do partograma } \\
\hline Não preenchido & 7 & 4,46 \\
\hline Incompleto & 18 & 11,47 \\
\hline Completo & 25 & 15,92 \\
\hline Não encontrado & 107 & 68,15 \\
\hline \multicolumn{3}{|c|}{ Utilização da episiotomia } \\
\hline Sim & 73 & 46,50 \\
\hline Não & 75 & 47,77 \\
\hline Não registrado & 9 & 5,73 \\
\hline \multicolumn{3}{|c|}{ Manobras ativas $-3^{\circ}$ est. } \\
\hline Sim & 110 & 70,06 \\
\hline Não & - & - \\
\hline Não registrado & 47 & 29,94 \\
\hline \multicolumn{3}{|c|}{ Realização da amniotomia } \\
\hline Sim & 7 & 4,45 \\
\hline Não & - & - \\
\hline Não registrado & 150 & 95,55 \\
\hline \multicolumn{3}{|l|}{ Uso de ocitocina } \\
\hline Sim & 127 & 80,90 \\
\hline Não & - & - \\
\hline Não registrado & 30 & 19,10 \\
\hline \multicolumn{3}{|c|}{ Contato pele a pele após o nascimento } \\
\hline Sim & 5 & 3,19 \\
\hline Não & - & - \\
\hline Não registrado & 152 & 96,81 \\
\hline \multicolumn{3}{|c|}{ Métodos não farmacológicos para alívio da dor } \\
\hline Banho de chuveiro & 1 & 0,64 \\
\hline Massagem & 1 & 0,64 \\
\hline Toques & - & - \\
\hline Outros & 5 & 3,18 \\
\hline Não registrados & 150 & 95,54 \\
\hline \multicolumn{3}{|l|}{ Local do parto } \\
\hline Triagem & - & - \\
\hline Pré-parto & - & - \\
\hline Sala de parto & 148 & 94,26 \\
\hline Não registrado & 9 & 5,74 \\
\hline
\end{tabular}

Tabela IV - Características maternas e perinatais das adolescentes gestantes. Maceió-AL, 2013.

\begin{tabular}{lcc}
\hline Características & n & \% \\
\hline Complicações clínicas ou obstétricas ocorridas no parto e puerpério & & \\
Sim & 21 & 13,37 \\
Não & 136 & 86,63 \\
Não registrado & 0 & 0 \\
Tempo de permanência na instituição da internação até a alta & 37 & 23,56 \\
Horas mãe & 117 & 74,53 \\
Dias mãe & 3 & 1,91 \\
Não registrado & & 71,34 \\
Destino do RN & 112 & 10,83 \\
Alcon & 17 & 17,20 \\
UCI neonatal & 27 & 0 \\
UTI neonatal & 0 & 0,63 \\
Necrotério & 1 & \\
Não registrado & 17 \\
\hline
\end{tabular}

Alcon: Alojamento conjunto; UCI: Unidade de cuidados intensivos; UTI: Unidade de terapia intensiva. 


\section{DISCUSSÃO}

O presente estudo mostra o perfil do atendimento a adolescentes gestantes em uma maternidade pública do estado de Alagoas e indica ainda elevada prevalência de partos nessa faixa etária, apesar de o Ministério da Saúde (MS) afirmar uma redução de $30 \%$ no número de partos em adolescentes na última década ${ }^{(5)}$.

Entretanto, na amostra avaliada foi encontrada prevalência de gestantes adolescentes provenientes do interior do estado. Porém, esses achados são apoiados em dados do IBGE, os quais indicam que as regiões Norte e Nordeste apresentam as mais altas taxas de fecundidade do país, em especial nas áreas rurais ${ }^{(15)}$. Esse achado apresenta aspectos sociais, culturais e econômicos como determinantes dessa gravidez precoce, pois nas regiões Norte e Nordeste, em especial nas cidades menores, o acesso à informação é mais escasso e o esclarecimento da população é limitado.

Tratando-se do estado civil, o estudo evidenciou que grande parte das parturientes adolescentes pesquisadas vive em união estável. Resultado semelhante foi encontrado em um estudo realizado em Indaiatuba-SP ${ }^{(16)}$, onde a maioria $(78,8 \%)$ das adolescentes também mantém união estável após a gestação. Em outro estudo, desenvolvido em Fortaleza- $\mathrm{CE}^{(17)}$, a maioria das adolescentes afirmou se manter solteira ou em união estável, fato que reforça, para a sociedade, a ideia de relacionamento inconstante entre os adolescentes $^{(17)}$.

A ocorrência elevada de adolescentes em união estável está ligada a uma satisfação imposta pela sociedade em se formar uma família, mesmo que temporária, a fim de estruturar a formação do futuro filho ${ }^{(18)}$. Além disso, nas uniões entre adolescentes, há grande risco de união com outros parceiros, e isso aumenta em $40 \%$ a possibilidade de uma nova gestação. Consequentemente, quando a adolescente está morando com o companheiro, as chances de uma nova gravidez indesejada são reduzidas ${ }^{(19)}$.

Quanto ao grau de escolaridade, observou-se que as parturientes adolescentes tiveram de 4 a 7 anos de estudo, demonstrando uma baixa escolaridade. Esse abono escolar pode estar associado à gravidez, somada à vergonha dos colegas e ao fato de assumirem os cuidados com o $\operatorname{lar}^{(20)}$.

Da mesma forma, estudos desenvolvidos em outros estados, como Bahia, Rio de Janeiro e Rio Grande do Sul, mostraram que, das adolescentes grávidas, 35,3\% tinham apenas o ensino fundamental incompleto e 44,1\% possuíam o ensino médio incompleto ${ }^{(21)}$. Com isso, a probabilidade de as mães adolescentes tornarem-se adultas economicamente bem sucedidas é menor, devido ao baixo nível de desempenho escolar ${ }^{(20)}$.

Ao analisar o número de gestações e partos, que são importantes dados obstétricos, observa-se que a amostra apresenta maior proporção nos números de parto em comparação aos números de gestação. Isso ocorre porque o aborto foi considerado uma gestação como as outras.

Nota-se, com isso, que as parturientes adolescentes estavam em sua primeira gestação. Resultado semelhante foi encontrado em uma pesquisa realizada em TeresinaPI, onde $69,5 \%$ das adolescentes eram primigestas ${ }^{(3)}$. Em estudo análogo, realizado no município de Catanduva-SP, a média de idade das adolescentes ao dar à luz pela primeira vez foi de 15 anos, enquanto a média da segunda gestação foi de 17 anos $^{(6)}$. Esses resultados são corroborados por outros autores, que destacam, inclusive, a existência de correlação entre reincidência de gravidez, maior número de filhos e idade da primeira gravidez, a qual, por sua vez, está relacionada com o início precoce das atividades sexuais $^{(6,11,16)}$.

A respeito da realização de consultas no pré-natal, grande parte das adolescentes realizou de 4 a 6 consultas. De acordo com o Ministério da Saúde, o número adequado de consultas no pré-natal seria igual ou superior a seis, sendo mensais até a $28^{\mathrm{a}}$ semana, quinzenais entre 28 e 36 semanas, e semanais no termo ${ }^{(5)}$.

Com relação às boas práticas obstétricas utilizadas nas parturientes adolescentes, o partograma não foi encontrado na maioria dos prontuários avaliados, sendo resultado semelhante identificado em um estudo sobre atenção humanizada ao parto de adolescente, em que a utilização do partograma foi evidenciada em apenas um terço dos prontuários analisados ${ }^{(8)}$. Assim, este estudo apresenta resultados negativos relacionados à atenção durante a evolução do parto das adolescentes, visto que a Organização Mundial de Saúde (OMS) recomenda o uso do partograma nas maternidades para o acompanhamento da evolução do trabalho de parto, evitando complicações que possam terminar em óbito materno e neonatal.

A redução dos registros quanto à realização da amniotomia dificultou a avaliação dessa prática. Em comparação, dois estudos distintos, realizados no Rio Grande do Sul, apontaram que a amniotomia foi realizada demasiadamente ${ }^{(8,9)}$. Embora existam evidências de que a amniotomia precoce possa reduzir a duração do trabalho de parto, efeitos indesejáveis podem ocorrer, como aumento do risco de uma operação cesariana, prolapso de cordão umbilical, compressão do cordão com aumento da frequência de desacelerações fetais e hemorragias, e infecções ascendentes. Por isso, a amniotomia deve ser evitada, devendo ser realizada apenas durante o trabalho de parto, com indicações clínicas específicas ${ }^{(19-20)}$.

Quanto à utilização de ocitocina nos três estágios do parto, observou-se que essa prática foi utilizada, em sua maior parte, no terceiro estágio. Esse é um dado positivo em relação a outro estudo, realizado em Pelotas-RS, no 
qual a administração de ocitocina no primeiro estágio foi de $91,7 \%^{(9)}$. O uso de ocitocina deve ser feito com vigilância do bem-estar materno e fetal. As rotinas que levam à medicalização desnecessária da mulher durante o trabalho de parto devem ser repensadas, uma vez que não existem benefícios comprovados no uso rotineiro de ocitocina, mas existem efeitos colaterais, tais como a hiperestimulação uterina e o aumento da dor ${ }^{(21)}$.

No que se refere ao contato pele a pele entre mãe e filho, percebeu-se que na maioria das vezes essa prática não foi estimulada ou não foi registrada nos prontuários pelos profissionais. Resultado semelhante foi encontrado em uma pesquisa desenvolvida em Pelotas-RS, na qual o contato precoce mãe/bebe nem sempre foi incitado e a maior parte dos profissionais não estimulava a parturiente a amamentar seu filho ${ }^{(9)}$. Segundo o Ministério da Saúde, são vários os benefícios relacionados à amamentação iniciada logo após o parto, entre eles, a indução da contração uterina, estimulada pela liberação de ocitocina endógena através da sucção, o que pode reduzir o sangramento materno depois do nascimento ${ }^{(5)}$.

Um estudo sobre o uso de métodos não farmacológicos para o alívio da dor e sua considerável eficácia informou que a posição, frequência de mudanças de posição e deambulação exercem efeitos profundos sobre a atividade e a eficácia uterina. O uso da bola de parto permite a mudança de posição, diminuindo a sensação dolorosa da contração uterina, estimula movimentos espontâneos e não habituais, e ajuda na rotação e descida fetal. Já as técnicas respiratórias e de relaxamentos, assim como o banho de chuveiro, trouxeram outra forma de combater as dores do parto, além de reduzir a ansiedade e tensão muscular ${ }^{(22)}$.

Com relação às complicações durante o parto, observouse que as parturientes adolescentes não tiveram nenhum tipo de complicação. Considerando que a sala de parto faz parte do centro obstétrico, observa-se que a maioria dos partos ocorreu na sala de parto. Sendo assim, a qualidade da assistência ao parto depende de componentes estruturais e funcionais do Centro Obstétrico $(\mathrm{CO})^{(22)}$. Nesse sentido, a assistência à parturiente adolescente mostrou-se adequada, visto que a maioria teve seus filhos na sala de parto.

Com ênfase no tempo de permanência na instituição, o Ministério da Saúde publicou, em setembro de 1993, uma portaria enfatizando que as altas não deveriam ser dadas antes de 48 horas, por considerar importante o alto teor educativo inerente ao sistema de alojamento conjunto, e por ser esse período importante na detecção de patologias neonatais ${ }^{(5)}$.

Alguns dados da pesquisa apresentaram-se limitados devido à carência dos registros nos prontuários, o que implicou na avaliação da assistência voltada para a adolescente parturiente.

\section{CONCLUSÃO}

Evidenciou-se com o estudo que algumas práticas obstétricas preconizadas pelo Ministério da Saúde estão sendo realizadas com as parturientes adolescentes, mas não são suficientes para uma assistência de qualidade. Recomenda-se que os profissionais sejam sensibilizados quanto à importância da assistência humanizada, sendo capacitados e qualificados para prestarem esse serviço.

\section{REFERÊNCIAS:}

1. Melo JS, Kerber NPC, Oliveira AMN, Busanello J, Silveira TS, Pohlmann FC. Inserção do acompanhante no cuidado da adolescente em um centro obstétrico do sul do país. Ciênc Cuid Saúde. 2011;10(4):781-8.

2. Ministério da Saúde (BR), Secretaria de Atenção à Saúde, Área de Saúde do Adolescente e do Jovem. Marco legal: saúde, um direito de adolescentes. Brasília: Ministério da Saúde; 2005.

3. Vilarinho LM, Nogueira LT, Nagahama EEI. Avaliação da qualidade da atenção ao trabalho de parto e parto de adolescentes. Rev Enferm UERJ. 2013;21(2):221-7.

4. World Health Organization - WHO. Pregnant adolescents: delivering on global promises of hope. Geneva: WHO; 2006.

5. Ministério da Saúde (BR), Secretaria de Atenção à Saúde, Departamento de Atenção Básica. Atenção ao pré-natal de baixo risco. Brasília: Ministério da Saúde; 2012. (Série A. Normas e Manuais Técnicos. Cadernos de Atenção Básica, n. 32.)

6. Manfredo VA, Cano MAT, Santos BMO. Reincidência de gravidez em adolescentes: retrato de uma realidade. Rev APS. 2012;15(2):192-8.

7. Ministério da Saúde (BR), Secretaria de Atenção à Saúde, Departamento de Ações Programáticas Estratégicas. Gestação de alto risco: manual técnico. $5^{\mathrm{a}}$ ed. Brasília: Ministério da Saúde; 2010.

8. Busanello J, Kerber NPC, Mendoza-Sassi RA, Mano PS, Susin LRO, Gonçalves BG. Atenção humanizada ao parto de adolescentes: análise das práticas desenvolvidas em um Centro Obstétrico. Rev Bras Enferm. 2011;64(5):824-32.

9. Silva RC, Soares MC, Jardim VMR, Kerber NPC, Meincke SMK. O discurso e a prática do parto humanizado de adolescentes. Texto \& Contexto Enferm. 2013;22(3):629-36.

10. Molina RC, Roca CG, Zamorano JS, Araya EG. Family planning and adolescente pregnancy. Best Pract Res Clin Obstet Gynaecol. 2010;24(2):209-22. 
11. Silva ACA, Andrade MS, Silva RS, Evangelista TJ, Bittencourt IS, Paixão GPN. Fatores de risco que contribuem para a ocorrência da gravidez na adolescência: revisão integrativa da literatura. Rev Cuid (Bucaramanga). 2013;4(1):531-9.

12. Busanello J, Kerber NPC, Lunardi Filho WD, Lunardi VL, Sassi-Mendoza RA, Azambuja EP. Parto humanizado de adolescentes: concepção dos trabalhadores da saúde. Rev Enferm UERJ. 2011; 19(2):218-23.

13. Enderle CF, Kerber, NPC, Susin LO, Goncalves BG. Parto de adolescentes: elementos qualitativos da assistência. Rev Esc Enferm USP. 2012;46(2):287-94

14. Ministério da Saúde (BR), Secretaria de Atenção em Saúde, Departamento de Ações Programáticas Estratégicas. Diretrizes nacionais para a atenção integral à saúde de adolescentes e jovens na promoção, proteção e recuperação da saúde. Brasília: Ministério da Saúde; 2010.

15. Garcia SAL, Lippi UG, Garcia SAL. O parto assistido por enfermeira obstetra: Perspectivas e controvérsias. Rev Bras Promoç Saúde. 2010;23(4):380- 8.

16. Santos JO, Silva CFS, Petenão E, Soster FCB, Berard $\mathrm{MB}$, Silva SR. Perfil das adolescentes com reincidência de gravidez assistidas no setor público de Indaiatuba (SP). Rev Inst Ciênc Saúde. 2009;27(2):115-21.

17. Rodrigues FRA, Rodrigues DP, Souza ES, Nogueira MEF, Fialho AVM. A vivência do ciclo gravídicopuerperal na adolescência: perfil sociodemográfico e obstétrico. REME Rev Min Enferm. 2008;12(1):27-33.

18. Nery IS, Gomes KRO, Barros IC, Fernandes ACN, Gomes IS, Viana LMM. Gravidez na adolescência: fatores preditores da reincidência. In: Nery ID, Gomes KRO, Barros IC, Viana LMM. Gravidez na adolescência: prevenção e risco. Teresina: EDUFPI; 2011. p. 25.

19. Bruno ZV, Feitosa FEL, Silveira KP, Morais IQ, Bezerra MF. Reincidência de gravidez em adolescentes. Rev Bras Ginecol Obstet. 2009;31(10):480-4.

20. Martins MDG, Santos GHND, Sousa MDS, Costa JEFBD, Simões VMF. Associação de gravidez na adolescência e prematuridade. Rev Bras Ginecol Obstet. 2011;033(11):354-60.

21. Viellas EF, Gama SGN, Theme Filha MME, Leal MC. Gravidez recorrente na adolescência e os desfechos negativos no recém-nascido: um estudo no Município do Rio de Janeiro. Rev Bras Epidemiol. 2012;15(3):443-54.

22. Sescato AC, Souza SRRK, Wall ML. Os cuidados não-farmacológicos para alívio da dor no trabalho de parto: orientações da equipe de enfermagem. Cogitare Enferm. 2008; 13(4):880-8.

\section{Endereço do primeiro autor:}

Márcia Juliana Mello da Silva

Escola de Enfermagem e Farmácia da Universidade Federal de Alagoas

Av. Lourival Melo Mota, s/n

Bairro: Tabuleiro dos Martins

CEP: 57072-900 - Maceió - AL - Brasil

Email: jullyanna_ms@hotmail.com

\section{Endereço para correspondência:}

Amuzza Aylla Pereira dos Santos

Rua Durval Guimarães 1354

Bairro: Ponte Verde

CEP: 57035-060 - Maceió - AL - Brasil

E-mail: amuzzasantos@bol.com.br 